\title{
NONLINEAR OPTICAL IMAGING WITH SUB-10 FS PULSES
}

Marcos Dantus ${ }^{*}$, Dmitry Pestov, and Yair Andegeko

Department of Chemistry, Michigan State University, East Lansing MI, 48824

*Corresponding author: dantus@msu.edu

\section{Introduction}

The fundamental goal of biomedical imaging is the retrieval of structural and dynamical information from the sample, with the greatest contrast, spatial and temporal resolution possible. The workhorse of biomedical imaging is an optical microscope, which readily provides the resolution sufficient to observe sub-cellular organelles. The introduction of lasers and confocal optics brought the resolution scale down to diffraction-limited, i.e. on the order of the laser wavelength. Contrast enhancement came primarily from the use of fluorescent labeling compounds.

It is well-known that at high enough electric field intensities, achieved usually by focusing the laser radiation, a number of nonlinear processes can come into play. However, it was not recognized until recently that the nonlinear optical processes can be used to attain the resolution well beyond the diffraction limit (Dyba and Hell 2002; Westphal and Hell 2005). The nonlinear effects have also permitted imaging through scattering biological tissue. In this chapter, we concentrate on two-photon microscopy (TPM) and explore the advantages and disadvantages of using ultrashort $<10$ fs laser pulses for this purpose.

TPM was introduced at a time when advanced optical microscopy involved the use of multiple 
laser wavelengths (Denk, Strickler, and Webb 1990). For a given sample, one would need to swap optics in order to excite different fluorescent labels and thus achieve the greatest contrast. With TPM, it became possible to excite a whole range of different laser dyes without having to change optics. Because of the quadratic dependence of the two-photon excitation fluorescence (TPEF) signal on the laser peak intensity, the use of two-photon-resonant processes reduced the interaction region to a small volume of the sample where the laser light was focused. It also allowed one to image as far as one millimeter through scattering tissue (Theer, Hasan, and Denk 2003).

It is known that with the laser pulse energy kept constant, the TPEF signal magnitude is inversely proportional to the laser pulse duration. This is because the total amount of the collected signal not only depends on the peak field intensity but also on the time duration that the laser field is acting upon the sample. The availability of femtosecond lasers, and in particular, Ti:Sapphire lasers (Spence, Kean, and Sibbett 1991), fostered the development of TPM but not for long. When TPM was introduced, the available laser sources produced 150-fs pulses. Now, in 2008 , there are a number of companies offering lasers capable of generating sub-10 fs pulses.

Despite the promise of 15 -fold brighter signals, these new laser systems are not being used for TPM. Here we discuss reasons why and present a method which makes the utilization of these ultrashort pulse sources possible.

\section{Pulse Dispersion}

When femtosecond pulses propagate through an optical medium they undergo chromatic dispersion. The cause for dispersion is that all optical media have a frequency-dependent index 
of refraction. Because of the uncertainty principle, femtosecond pulses have broad spectra, and this makes them orders of magnitude more sensitive to chromatic dispersion when compared to nano- or even picosecond pulses. If the phase retardation introduced by the medium is expanded in a Taylor series about the carrier frequency, the term that changes linearly with the frequency shift can be associated with a time delay and therefore, has no effect on the pulse shape and the TPM output. The subsequent terms, however, do affect the performance of TPM. The term which is quadratic in frequency is due to the second-order dispersion (SOD), also known as group delay dispersion (GDD). By definition, GDD is the derivative of the group delay with respect to the angular frequency, or the second derivative of the spectral phase. It is usually specified in $\mathrm{fs}^{2}$ or $\mathrm{ps}^{2}$. The GDD per unit length (in units of $\mathrm{s}^{2} / \mathrm{m}$ ) is refereed to as group velocity dispersion (GVD).

If we consider a femtosecond pulse free of any phase distortion, such a pulse would satisfy the transform limit (TL) equation, which for Gaussian pulses is given by $\Delta v \times \Delta t=2 \ln (2) / \pi$ with $\Delta v$ and $\Delta t$ being the $1 /$ e spectral bandwidth (in $\mathrm{Hz}$ ) and the time duration of the pulse. When such a pulse acquires positive GDD, the high-frequency (blue-shifted) components are delayed with respect to the low-frequency (red-shifted) ones, and the pulse gets broadened by a factor of $\left(1+\left(4 \ln (2) \phi^{\prime \prime} / \tau_{\text {in }}^{2}\right)^{2}\right)^{1 / 2}$, where $\phi^{\prime \prime}$ is the amount of GDD, and $\tau_{\text {in }}$ is the input pulse duration. The effect of negative GDD is the same, except for now the high-frequency components run ahead of low-frequency ones, see Fig. 1. SOD lowers the peak intensity of the pulses and hence reduces the two-photon excitation efficiency.

The term that depends on the third power of the frequency shift is known as third-order 
dispersion (TOD). When a TL pulse acquires a significant amount of TOD, the pulse envelope is distorted and a series of sub-pulses is produced, as shown in Fig. 1. Unlike a pulse with SOD, a pulse with TOD leads to two-photon excitation with the same efficiency as a TL pulse but only for a particular two-photon frequency. At other frequencies, the amount of excitation is suppressed. The control over TOD would allow for preferential excitation in different spectral regions, while its correction would lead to efficient two-photon excitation over the whole accessed spectral range. Unfortunately, measuring and correcting TOD is not a simple task.

The reason why two-photon excitation efficiency depends on the spectral phase of the laser pulse is beyond the scope of the peak intensity argument given above. It requires a more detailed consideration of how the pulse contributes to the nonlinear optical transitions. Let us consider, e.g., the case of two-photon excitation at wavelengths corresponding to $\lambda_{\text {TPE }}=400 \mathrm{~nm}$. In addition to a pair of 800 -nm photons, there are multiple combinations of spectral components within the bandwidth of an ultrashort pulse that can combine to cause excitation equivalent to $400 \mathrm{~nm}$. Their wavelengths satisfy the relation $1 / \lambda_{\text {long }}+1 / \lambda_{\text {short }}=1 / \lambda_{\text {TPE }}$. In particular, one can have photons at $790 \mathrm{~nm}$ combining with photons at $810 \mathrm{~nm}$. Obviously, these channels will interfere constructively only if they all have the same phase. Mathematically, the requirement can be expressed as $\phi_{\text {long }}+\phi_{\text {short }}=$ const, i.e. the sum of the phases of the longer- and shorterwavelength photons is independent of the choice of the wavelength pair. This condition is satisfied when all spectral components of the laser pulse have the same phase, i.e. for a TL pulse. Another interesting situation is when the sum is constant (e.g., zero) only for one specific value of $\lambda_{\text {TPE }}$. This can be accomplished by using a phase function that is anti-symmetric with respect to $\lambda_{\text {TPE }} / 2$. This arrangement will be discussed later within the context of selective two-photon 
excitation. For pure TOD, an anti-symmetric function, the condition above is satisfied for only one $\lambda_{\text {TPE }}$ value for which TPE efficiency is maximum, at other wavelengths TPE efficiency is reduced.

\section{Measuring and Eliminating Pulse Dispersion}

The main source of dispersion in microscopy is the microscope objective lens. Therefore, the first step is to obtain an accurate measurement of the spectral phase at the focal plane; the second step is to use adaptive optics to compensate the measured phase. A method that incorporates the indicated steps was invented by our research group in 2003 (Pastirk et al. 2003; Lozovoy, Pastirk, and Dantus 2004; Xu et al. 2006; Dantus, Lozovoy, and Pastirk 2007). This method, known as multiphoton intrapulse interference phase scan (MIIPS), uses a pulse shaper with a programmable spatial light modulator to introduce a reference phase function $f(\lambda)$ that causes cancellation of the linear chirp at some wavelengths within the pulse spectrum. At those wavelengths, yet unknown, nonlinear processes such as second harmonic generation (SHG) are maximized. MIIPS uses this information to solve the equation $\phi^{\prime \prime}(\lambda)-f^{\prime \prime}(\lambda)=0$ and find the second derivative of the original phase $\phi(\lambda)$ and its corresponding wavelength (Lozovoy et al. 2008). When the difference is zero, $f^{\prime \prime}(\lambda)$ is exactly the second derivative of the phase distortion to be measured. After double integration of the retrieved $\phi^{\prime \prime}(\lambda)$, the chromatic dispersion at the focal plane is known and the first step is complete. Note that the measured $\phi^{\prime \prime}(\lambda)$ is a function of wavelength, not a constant in the Taylor series expansion, that contains information about highorder phase distortions including SOD, TOD and beyond. For the second step, the pulse shaper introduces a phase function that cancels the measured phase distortions at all wavelengths. This 
results in TL pulses at the focal plane. This process is fully automated and can be completed in less than one minute.

The ability to cancel all orders of phase distortion gives us an opportunity to evaluate the effect of partial dispersion correction on TPM. In particular, we focus on comparing SOD correction, which can be achieved with a prism pair arrangement, and correction of all orders of phase dispersion using MIIPS. For these measurements we used a pair of prisms in addition to our pulse shaper. With the aid of the pulse shaper, we found the condition for which SOD at the center wavelength was fully eliminated by the prism pair, and only higher-order dispersion was compensated by the pulse shaper.

As a first experiment, we wanted to return to the fundamental premise of TPM, which states that the efficiency of the two-photon excitation should be inversely proportional to the pulse duration. For TL pulses it is equivalent to having two-photon excitation efficiency proportional to the spectral bandwidth of the laser pulses.

The measured intensities of TPEF as a function of the spectral bandwidth, for GDD-compensated and TL pulses, are shown in Fig. 2. The experiments were performed with the same average power at the sample $(2.3 \mathrm{~mW})$ for all bandwidths, using a red fluorescence standard slide (Chroma Technologies). We found that in the case of TL pulses, obtained via MIIPS compensation, the magnitude of TPEF increased linearly almost over the whole studied range. It was enhanced by a factor of 6 as the laser bandwidth was tuned from 10 to $80 \mathrm{~nm}$. In contrast, the laser pulses compensated only for SOD demonstrated a modest effect on the signal gain. 
Once the bandwidth reached $30 \mathrm{~nm}$, no further increase in TPEF was observed. Therefore, we conclude that above 30-nm bandwidth higher order dispersion, introduced by the prisms and the microscope objective, becomes dominant in the degradation of signal intensity in TPM.

\section{Compensated pulses results in higher signal in two-photon imaging of biological samples}

The results shown above are in agreement with theory. However, it is important to demonstrate that they are applicable towards actual TPM of biological samples. We show here the effect of dispersion compensation on fixed biological samples and in living cells. TPM images on a fixed sample of mouse intestine (Molecular Probes, F-24631), given in Fig. 3, were obtained with GDD-only compensation and after full MIIPS compensation for high-order dispersion. The average increase in the TPEF signal between these two conditions was $\sim 5$ fold. Figures obtained for GDD-only compensation are shown as obtained and also after a digital 6x increase in intensity. The increased two-photon efficiency achieved by using MIIPS results in an improvement of signal-to-noise ratio and contrast.

The next step was to test the difference between SOD compensation and compensation for highorder dispersion using MIIPS on living cells. Living U2OS cells stained with nuclear (blue) or mitochondrial (green) stain were imaged using GDD-only and MIIPS compensated pulses, as shown in Fig. 4. The average increase in signal was $\sim 5$ fold, similar to the results shown in Fig 3. The observed increase of the excitation efficiency can be used to attenuate the incident laser power while maintaining satisfactory signal level. This attenuation would then reduce the exposure of the cells to the deleterious effects of the laser. We explore this effect later in the 
chapter.

\section{Two-photon depth imaging}

One of the most important advantages of TPM over other microscopy modalities is the ability to image deep within biological tissue. Among the most impressive results we highlight the article by Denk and coworkers where they imaged neurons at a depth exceeding $1000 \mu \mathrm{m}$ (Theer, Hasan, and Denk 2003). Based on the increases in signal that we have registered through the use of dispersion compensated ultrashort pulses, we evaluated the effects of the increased signal on depth imaging. The motivation for this exploration is that deep tissue imaging will be essential to unravel biologically complex phenomena such as cancer. For example, the initial escape of metastatic cells from tumors has eluded studies in vivo, partly because of the relative inaccessibility of this process to direct observation. Recently, multi-photon microscopy has been applied to intravital-imaging studies (Peti-Peterdi et al. 2002; Sandoval et al. 2004; Molitoris and Sandoval 2005; Konig et al. 2007) and has been especially useful for characterizing primarytumor properties, growth rates and mechanisms of metastasis to target organs. Animal models of cancer that use the stable expression of green fluorescent protein (GFP) from tissue specific promoters now make it possible to directly observe cell behavior in primary tumors of live animals, without worries that the labeling agent is not evenly taken up by the cells.

It is important to realize that dispersion compensation can eliminate the high-order phase distortions (in the spectral domain) introduced by the objective lens, as discussed above, but it cannot eliminate the scattering (in the spatial domain) that occurs in depth imaging. Here we explore the use of laser pulses that are dispersion compensated only before the medium. In 
principle, it is possible to compensate for dispersion at greater depths but if the dispersion of tissues is similar to that of pure water, it should be insignificant. Finally, we could titrate the amount of laser power used, increasing the intensity as the focal plane moves deeper into the tissue.

To study the implications of spectral phase correction for two-photon depth-resolved imaging, we imaged a thick section of mouse kidney tissue, stained with DAPI (cell nuclei), Mitotracker488 (mitochondria), and Phalloidin-568 (actin). The results, summarized in Fig. 4, demonstrate increased penetration depth when higher-order dispersion is compensated via MIIPS, compared to GDD-only compensation. The images show the collagen wall components of a blood vessel in the mouse kidney at a depth of $40 \mu \mathrm{m}$. The collection duct region above it, at a depth of $50 \mu \mathrm{m}$, is seen only when MIIPS is applied.

The results from the depth-resolved imaging strongly support our hypothesis that dispersion compensation, especially when using pulses shorter than $20 \mathrm{fs}$, can make a very significant difference. Five-fold increase in the signal magnitude is typical, and some locations exhibit signal enhancement by as much as an order of magnitude.

\section{Effect of dispersion compensation on photobleaching}

The results shown in Figs. 3-5 indicate that the use of ultrashort, dispersion-compensated pulses can lead to essential improvements in TPM. However, one still needs to determine if those improvements are accompanied by a concomitant increase in photobleaching and other photoinduced damaging processes. Assuming that laser-induced effects are proportional to the amount 
of molecules that are excited by the laser pulse, greater signal would necessarily imply greater damage. Greater damage could also be caused by three-photon processes, which are expected to be more probable when using ultrashort TL pulses. Conversely, greater efficiency of two-photon excitation implies that a lower photon flux can be used, and this may reduce the damage. Minimizing photobleaching and photodamage is of great importance when imaging fixed samples, because each time the sample is imaged less signal is obtained. More importantly, when imaging living cells or imaging in-vivo tissues, photodamage can alter the biology that is being observed. For these reasons we proceeded to evaluate photobleaching induced by SOD compensated and by fully dispersion-compensated pulses using MIIPS.

For these experiments we picked a standard system that is easy to reproduce in other laboratories. We decided not to use a fluorescent dye solution because in microscopy, fluorescent tags are typically fixed. We also opted out a stained tissue as a sample since the experiment would be very difficult to reproduce by others. Based on these guidelines we decided to work with green fluorescent standard slides provided by Chroma Technologies. These slides are readily available to any microscopy laboratory and have the fluorophores evenly distributed within the sample. The only disadvantage is that the polymer matrix is different from a biological specimen, therefore our experiments will need to be validated in fixed tissue and living cell samples.

For the first set of experiments we used a laser intensity of $9 \mathrm{~mW}$ for both GDD-compensated and TL pulses. The resulting fluorescence signal profiles for the two cases are shown in Fig. 6 (black and grey solid circles, respectively). One can see that the use of TL pulses gives much 
higher magnitude of the TPEF signal but the change in photobleaching rates is subtle. We obtain somewhat more pronounced difference (Fig. 6, empty circles) after attenuation of the TL pulse energy by a factor of 3 so that the TPEF signal magnitude is about the same as for GDDcompensated pulses. In our earlier publication (Xi et al. 2008) all the three curves were fitted with a sum of four exponentials to retrieve the change in the relaxation rates. The inset in Fig. 6 is our attempt to use for data processing the phasor analysis (Digman et al. 2008).

The phasor method associates the decay dynamics with a vector in a so-called phasor space. In particular, purely exponential decay corresponds to a phasor with its end point on a semicircle of radius $1 / 2$ and centered at $(1 / 2,0)$. Tuning of the decay time from zero to infinity results in a counter-clockwise displacement of the end point from $(1,0)$ to $(0,0)$ along the semicircle. Multiexponential decay is equivalent to a point inside the semicircle but its dependence on the weightaveraged decay rate remains the same. Namely, a counter-clockwise shift around $(1 / 2,0)$ indicates an increase of the decay time (for details, see (Digman et al. 2008)). As one can see, the superposition of MIIPS compensation and lower pulse energies reduce the photobleaching rate. Let us note here though that the exposure time required for TPM imaging is much smaller than the measured photobleaching time. The laser-induced damage become an issue only for extensive real-time imaging experiments, when the sample is exposed to the laser radiation multiple times.

From the data shown in Fig. 6 we also learn two important aspects of photobleaching. First, there is a component of the process that depends on a higher nonlinear process, which we assume here to be instantaneous three-photon excitation. For high intensities, when dispersion is fully 
compensated, third-order processes become comparable to two-photon excitation and greater photobleaching is observed. Once the laser intensity is attenuated, three-photon processes are no longer as probable. Under these conditions the photobleaching process is not dependent on the absorption due to the aforementioned instantaneous three-three photon transitions but rather on two-photon excitation followed by excitation with an additional photon arriving 10-100 fs later. This sequential excitation process is possible because the excited state of the chromophore is long-lived compared with these delays. The greater efficiency of TL pulses allows the use of lower laser intensities and consequently reduces the damage the additional photons cause. For pulses compensated only for SOD, there are sub-pulses, as shown in Fig. 1, that can cause photodamage.

\section{Selective two-photon excitation}

As mentioned earlier, one of the great advantages of TPM was that a single laser could be used to excite a number of fluorescent molecules. However, this advantage is sometimes not desired. For example, there are several cases in which one would like to selectively excite one type and not all types of fluorescent molecules in the focal plane. One such situation would be the selective excitation of a particular fluorescent marker while preventing two-photon excitation of endogenous fluorescent molecules, which are abundant in living cells. Other situations could involve the determination of colocalization of different molecular species, and yet another application could be the selective activation of therapeutic photo-reagents while preventing damage to healthy tissue. Some of these problems are already being addressed by using newly available tunable femtosecond laser sources. Here we discuss an alternative approach. Using ultrashort pulses implies that the laser pulse bandwidth is ultra-broad. By phase or amplitude 
manipulation of the pulse spectrum we are able to achieve the equivalent function of tuning the laser pulse without actually changing any of the laser source parameters, thus preventing a change in optical alignment.

The simplest approach to implement selective two-photon excitation is to permit excitation only from a portion of the spectrum that is necessary for a specific fluorophore while blocking the portions that might cause excitation of different ones. For example, one could block longer or shorter wavelengths. To do this experimentally, one uses a pulse shaper, which is similar to a spectrometer. At the Fourier plane, the position where all the frequencies are spread and focused, one can alter the phase or amplitude of some frequencies without affecting others. The pulses are then reflected out of the device having acquired new spectral phase and amplitude properties. When the desired goal is to restrict excitation to shorter or longer wavelengths the undesired frequencies are simply blocked. However, if one desires highly selective two-photon excitation at one or more specific wavelengths, amplitude modulation leads to a significant loss of photons (Comstock et al. 2004). In other words, it is still effective but very inefficient.

Our first attempts at selective two-photon excitation made use of $\sim 10$ fs pulses modulated by a single sinusoidal phase function that stretched across the spectrum of the pulse (Pastirk et al. 2003; Dela Cruz, Pastirk, Lozovoy et al. 2004). These early attempts were far from optimum. Our group has since introduced an approach based on phase modulation to achieve highly selective nonlinear optical excitation (Comstock et al. 2004; Lozovoy et al. 2005; Lozovoy et al. 2006). This method introduces binary phase functions (using only 0 and $\pi$ values) to optimize two-photon excitation at the desired frequency while minimizing excitation elsewhere. When 
using binary phases, the resulting nonlinear fields, as all frequencies are coherently summed, acquire only two values, \pm 1 , making it relatively easy to maximize excitation at the desired frequency and minimize excitation elsewhere.

Following the successful demonstration of binary phase shaping for selective two-photon excitation in microscopy, we wanted to evaluate if this type of approach could be used for deep tissue imaging and also for selective photodynamic therapy (PDT). We wondered if the binary phase modulation would withstand the scattering introduced by the biological tissue. For these experiments we used as the imaging target three capillary tubes with a fluorescent $\mathrm{pH}$ sensitive dye, 8-hydroxypyrene-1,3,6-trisulfonic acid (HPTS), submerged in a cell containing the same dye at the same concentration. The $\mathrm{pH}$ in the capillary solution was acidic, and the $\mathrm{pH}$ in the surrounding solution was basic. In front of the sample cell, we placed a 1-mm slice of biological tissue (chicken breast muscle). When using TL pulses, it was impossible to discriminate between the two solutions (see Figs. 7A (no tissue) and 7B (with tissue)). We then designed a binary phase to cause excitation of the acidic solution, and a binary phase to cause excitation of the basic solution. The combination of these two excitation pulses was used to achieve selective functional imaging, shown in Figs. 7C (no tissue) and 7D (with tissue). The reason for the remarkable success of this experiment, reported in (Dela Cruz, Pastirk, Comstock et al. 2004), is that two-photon imaging relies on ballistic photons. Ballistic photons are those which undergo minimal (or none) scattering as they reach their target. Therefore, ballistic photons preserve the binary phase imposed for selective excitation. This implies that binary phase shaping can be used for deep tissue functional imaging and selective activation of photodynamic therapy agents. 
Selective two-photon excitation can provide additional contrast for single-cell imaging. It might be particularly useful for colocalization studies. In Fig. 8, it is illustrated on HeLa cells. The cells were co-stained with Phalloidin-350 (actin filaments), and with MitoTracker 488 (mitochondria). An amplitude shaping was used to get selective excitation of the two dyes by transmiting only lower (red) or higher (blue) part of the pulse spectrum in frequency domain, see Fig. 9. The actin filaments (blue) were visualized using the blue pulses, while the mitochondria (green) was imaged using red pulses. The image of a single HeLa cell in Fig. 8 clearly demonstrates typical sub-cellular localization of each structure.

\section{Conclusion}

We have identified high-order dispersion as the main reason why ultrashort, $\sim 10 \mathrm{fs}$, pulses have rarely been used for nonlinear optical imaging. We discussed the MIIPS method for automated measurement and elimination of high-order dispersion. We provided quantitative analysis for the advantage of high-order dispersion as compared with correction limited to SOD. This enhancement was confirmed experimentally in fixed and living cells, as well as in depth imaging. Finally, we demonstrated that the broad bandwidth of ultrashort pulses can be used for selective two-photon excitation when appropriate phase or amplitude modulation is used.

In summary, nonlinear optical imaging methods, such as TPM, allow for an order of improvement in the signal simply by utilization of 10 times shorter pulses. The enhancement is even more dramatic for higher-order nonlinearities, such as third-harmonic generation where two orders of magnitude increase is expected. These improvements, however, rely on successful measurement and elimination of phase distortions. 


\section{Acknowledgements}

We gratefully acknowledge funding for this research from the National Science Foundation, Major Research Instrumentation Grant CHE-0421047, and single investigator Grant CHE0500661. We thank Dr. Vadim Lozovoy for stimulating discussions and help with the phasor analysis, Dr. James H. Resau for technical assistance in the preparation of the mouse kidney sample. This work also benefited from the participation of Daniel Schlam through an undergraduate research opportunity. Some of the data was originally acquired by Lindsay R. Weisel, Dr. Peng Xi, and Dr. J. M. Dela Cruz. We are also grateful for the help from Nelson S. Winkler with the final preparation of the manuscript. 


\section{References}

Comstock, M., V. V. Lozovoy, I. Pastirk, and M. Dantus. 2004. Multiphoton intrapulse interference 6; binary phase shaping. Optics Express 12 (6):1061-1066.

Dantus, M., V. V. Lozovoy, and I. Pastirk. 2007. MIIPS characterizes and corrects femtosecond pulses. Laser Focus World 43 (5):101-104.

Dela Cruz, J. M., I. Pastirk, V. V. Lozovoy, K. A. Walowicz, and M. Dantus. 2004. Multiphoton intrapulse interference 3: Probing microscopic chemical environments. Journal of Physical Chemistry A 108 (1):53-58.

Dela Cruz, Johanna M., Igor Pastirk, Matthew Comstock, Vadim V. Lozovoy, and Marcos Dantus. 2004. Use of coherent control methods through scattering biological tissue to achieve functional imaging. Proceedings of the National Academy of Sciences 101 (49):16996-17001.

Denk, W., J. H. Strickler, and W. W. Webb. 1990. 2-Photon Laser Scanning Fluorescence Microscopy. Science 248 (4951):73-76.

Digman, M. A., V. R. Caiolfa, M. Zamai, and E. Gratton. 2008. The phasor approach to fluorescence lifetime imaging analysis. Biophysical Journal 94 (2):L14-L16.

Dyba, M., and S. W. Hell. 2002. Focal spots of size lambda/23 open up far-field florescence microscopy at $33 \mathrm{~nm}$ axial resolution. Physical Review Letters 88 (16):163901.

Konig, K., A. Ehlers, I. Riemann, S. Schenkl, R. Buckle, and M. Kaatz. 2007. Clinical twophoton microendoscopy. Microscopy Research and Technique 70 (5):398-402.

Lozovoy, V. V., I. Pastirk, and M. Dantus. 2004. Multiphoton intrapulse interference. IV. Ultrashort laser pulse spectral phase characterization and compensation. Optics Letters 29 (7):775-777.

Lozovoy, V. V., J. C. Shane, B. W. Xu, and M. Dantus. 2005. Spectral phase optimization of femtosecond laser pulses for narrow-band, low-background nonlinear spectroscopy. Optics Express 13 (26):10882-10887.

Lozovoy, V. V., B. Xu, Y. Coello, and M. Dantus. 2008. Direct measurement of spectral phase for ultrashort laser pulses. Optics Express 16 (2):592-597.

Lozovoy, V. V., B. W. Xu, J. C. Shane, and M. Dantus. 2006. Selective nonlinear optical excitation with pulses shaped by pseudorandom Galois fields. Physical Review A 74 (4):041805.

Molitoris, B. A., and R. M. Sandoval. 2005. Intravital multiphoton microscopy of dynamic renal processes. American Journal of Physiology-Renal Physiology 288 (6):F1084-F1089.

Pastirk, I., J. M. Dela Cruz, K. A. Walowicz, V. V. Lozovoy, and M. Dantus. 2003. Selective two-photon microscopy with shaped femtosecond pulses. Optics Express 11 (14):16951701.

Peti-Peterdi, J., S. Morishima, P. D. Bell, and Y. Okada. 2002. Two-photon excitation fluorescence imaging of the living juxtaglomerular apparatus. American Journal of Physiology-Renal Physiology 283 (1):F197-F201.

Sandoval, R. M., M. D. Kennedy, P. S. Low, and B. A. Molitoris. 2004. Uptake and trafficking of fluorescent conjugates of folic acid in intact kidney determined using intravital twophoton microscopy. American Journal of Physiology-Cell Physiology 287 (2):C517C526.

Spence, D. E., P. N. Kean, and W. Sibbett. 1991. 60-Fsec Pulse Generation from a Self-ModeLocked Ti-Sapphire Laser. Optics Letters 16 (1):42-44. 
Theer, P., M. T. Hasan, and W. Denk. 2003. Two-photon imaging to a depth of 1000 um in living brains by use of a Ti : Al2O3 regenerative amplifier. Optics Letters 28 (12):10221024.

Westphal, V., and S. W. Hell. 2005. Nanoscale resolution in the focal plane of an optical microscope. Physical Review Letters 94 (14):143903.

Xi, P., Y. Andegeko, L. R. Weisel, V. V. Lozovoy, and M. Dantus. 2008. Greater signal, increased depth, and less photobleaching in two-photon microscopy with $10 \mathrm{fs}$ pulses Optics Communications 281 (7):1841-1849.

Xu, B. W., J. M. Gunn, J. M. Dela Cruz, V. V. Lozovoy, and M. Dantus. 2006. Quantitative investigation of the multiphoton intrapulse interference phase scan method for simultaneous phase measurement and compensation of femtosecond laser pulses. Journal of the Optical Society of America B-Optical Physics 23 (4):750-759. 


\section{Figure Captions}

Fig. 1. Time-domain effects of the second and third order dispersion. A TL pulse is one that is as short as possible given the available bandwidth. GDD causes time separation between different wavelengths of the pulse, and this broadens its duration. Third order dispersion breaks the laser pulse into different sub-pulses in the time domain.

Fig. 2. Dependence of the TPEF signal on the laser spectral bandwidth for TL (filled squares) and SOD-compensated (open triangles) pulses. of the laser pulse. The linear relation between TPEF and bandwidth is observed only for TL, i.e. MIIPS-compensated, pulses. [The figure is adapted from (Xi et al. 2008). With permission.]

Fig. 3. Greater signal with MIIPS compensated pulses (TL) compared to GDD compensated pulses on mouse intestine tissue. Cross-section of fixed intestine, stained with Alexa Fluor 350 wheat germ agglutinin (goblet cells), phalloidin Alexa Fluor 568 phalloidin (actin) and SYTOX Green nucleic acid stain (nuclei). The image is of a surface area expansion of villus showing epithelial cells that cover the villi (top) and microvilli (bottom) in the small intestine. The image obtained using TL pulses had at least 5 times greater signal intensity than that taken with GDD compensated pulses. The intensity with GDD compensated pulses was amplified $6 \mathrm{x}$ for viewing image detail. Image size is $75 \mu \mathrm{m}$ (top) and $100 \mu \mathrm{m}$ (bottom). The objective used is Zeiss $40 \times$ LD, 1.1 NA.

Fig. 4. Greater signal with MIIPS compensated pulses compared to GDD compensated pulses is demonstrated in living U2OS cells. The cells were stained with Mito-Tracker 488 (mitochondria- 
green) and Hoechst 33258 (nuclei-blue). The image obtained using TL pulses had intensity of at least 5 times greater than that taken with GDD-only compensation. Image size is $100 \mu \mathrm{m}$ (top) and $50 \mu \mathrm{m}$ (bottom). The objective used is Zeiss $40 \times \mathrm{LD}, 1.1$ NA.

Fig. 5. Effect of dispersion compensation in two-photon depth imaging. Image acquisition of thick mouse kidney sample collected in a series of z-sections at different depths, which demonstrates that MIIPS compensated pulses attain better signal, and deeper penetration than those with GDD-only compensation. The image size is about $100 \mu \mathrm{m}$. The objective used is Nikon $60 \times 1.45 \mathrm{NA}$, with working distance of $130 \mu \mathrm{m}$. [The figure is adapted from (Xi et al. 2008). With permission.]

Fig. 6. Effect of dispersion and pulse energy on photobleaching. Photobleaching curves for GDD-only $(9 \mathrm{~mW})$ and MIIPS compensated $(9 \mathrm{~mW}$ and $3 \mathrm{~mW})$ pulses. Inset: Phasor plot for the three considered cases. The modulation frequency, which is a free parameter in these measurements (for details, refer to (Digman et al. 2008)), is taken to be $0.03 \mathrm{rad} / \mathrm{s}$. The counterclockwise shift of the phasor end point indicates the decrease of the decay rate.

Fig. 7. Selective two-photon imaging of HPTS. The sample consists of three 1-mm capillaries containing an acidic solution of HPTS submerged in a quartz cell filled with an alkaline solution of the same dye. To obtain the images, the sample was raster scanned (without and with scattering tissue) in the focal plane of the beam. (A and B) Two-photon excitation images obtained by raster scanning the sample without and with scattering tissue, respectively. The walls of the capillary tubes, which appear as vertical lines, are about $300 \mu \mathrm{m}$ thick and are clearly 
visible in both images. Note that comparable image quality is obtained in the presence and absence of biological tissue. The images shown in (A) and (B) were obtained with TL pulses, which do not allow discriminating between the two different $\mathrm{pH}$ solutions. (C) A functional image highlighting the contrast obtained by using coherent control. This image was produced by taking the ratio of the data acquired with two binary phase masks, chosen for selective excitation of different parts of the SHG spectrum. (D) The functional image obtained after a slice of biological tissue was placed in front of the sample. Note that the presence of the tissue reduces the overall signal-to-noise ratio, but the discrimination between acidic and alkaline HPTS is conserved. The contrast in the functional images can be further enhanced by using false color (C Upper and D Upper). Higher values are shown in red, and lower values are shown in blue. [The figure is adapted from (Dela Cruz, Pastirk, Comstock et al. 2004). With permission.]

Fig. 8. A section of a single HeLa (human cervical cancer) cell stained with Phalloidin 350 (actin filaments) and MitoTracker 488 (mitochondria) acquired using selective two-photon excitation, without fluorescence color filters. The image size is about $25 \mu \mathrm{m}$. [The figure is adapted from (Dantus, Lozovoy, and Pastirk 2007). With permission.]

Fig. 9. SHG spectra of the pulses used for selective excitation. [The figure is adapted from (Xi et al. 2008). With permission.] 


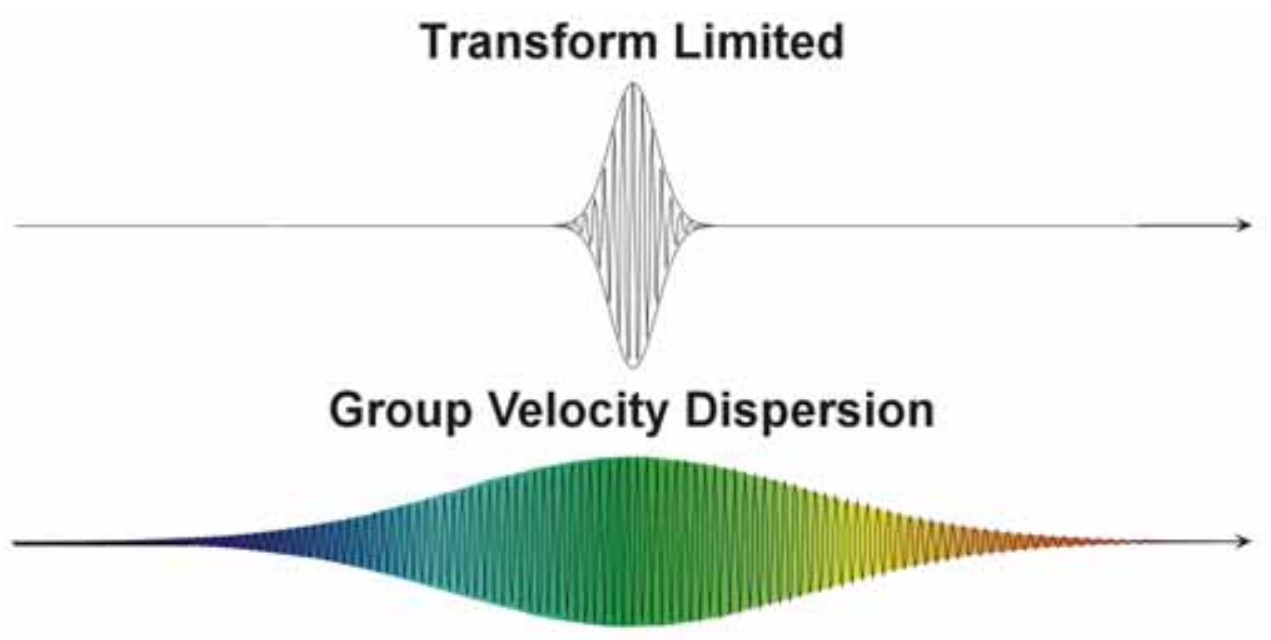

\section{Third Order Dispersion}

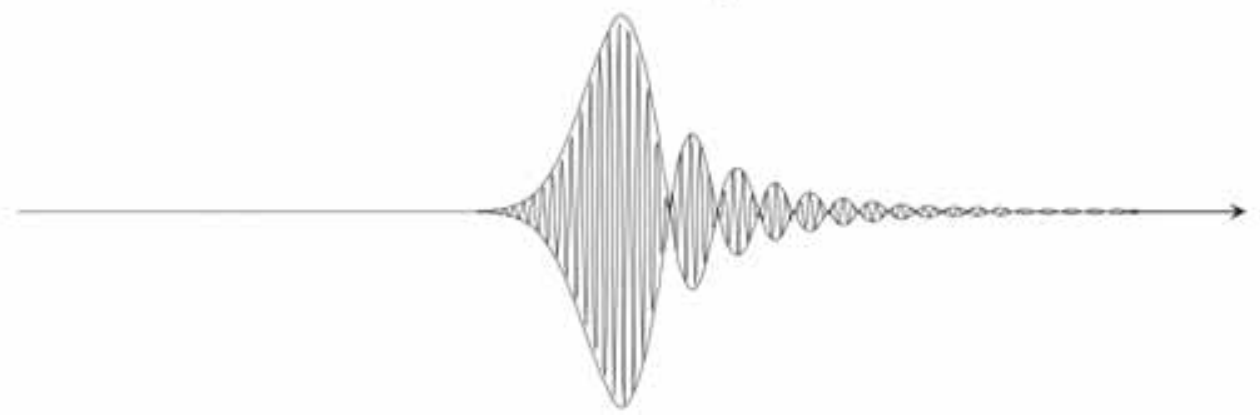

Fig. 1 


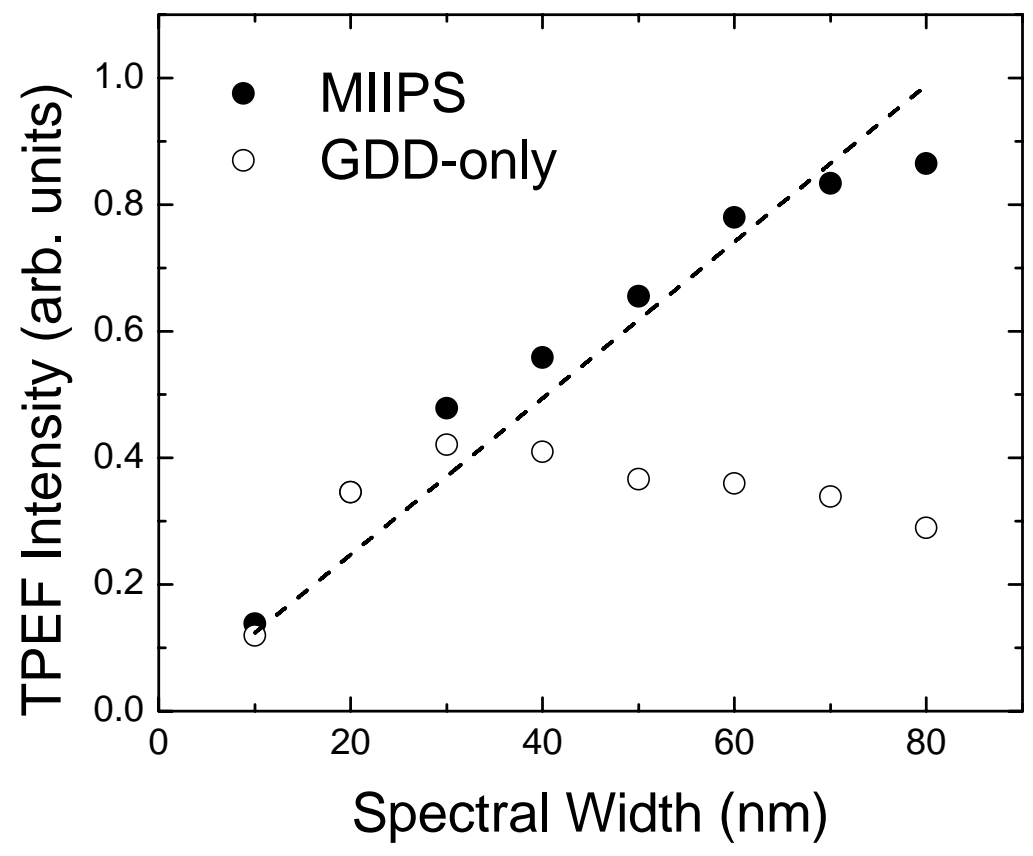

Fig. 2 


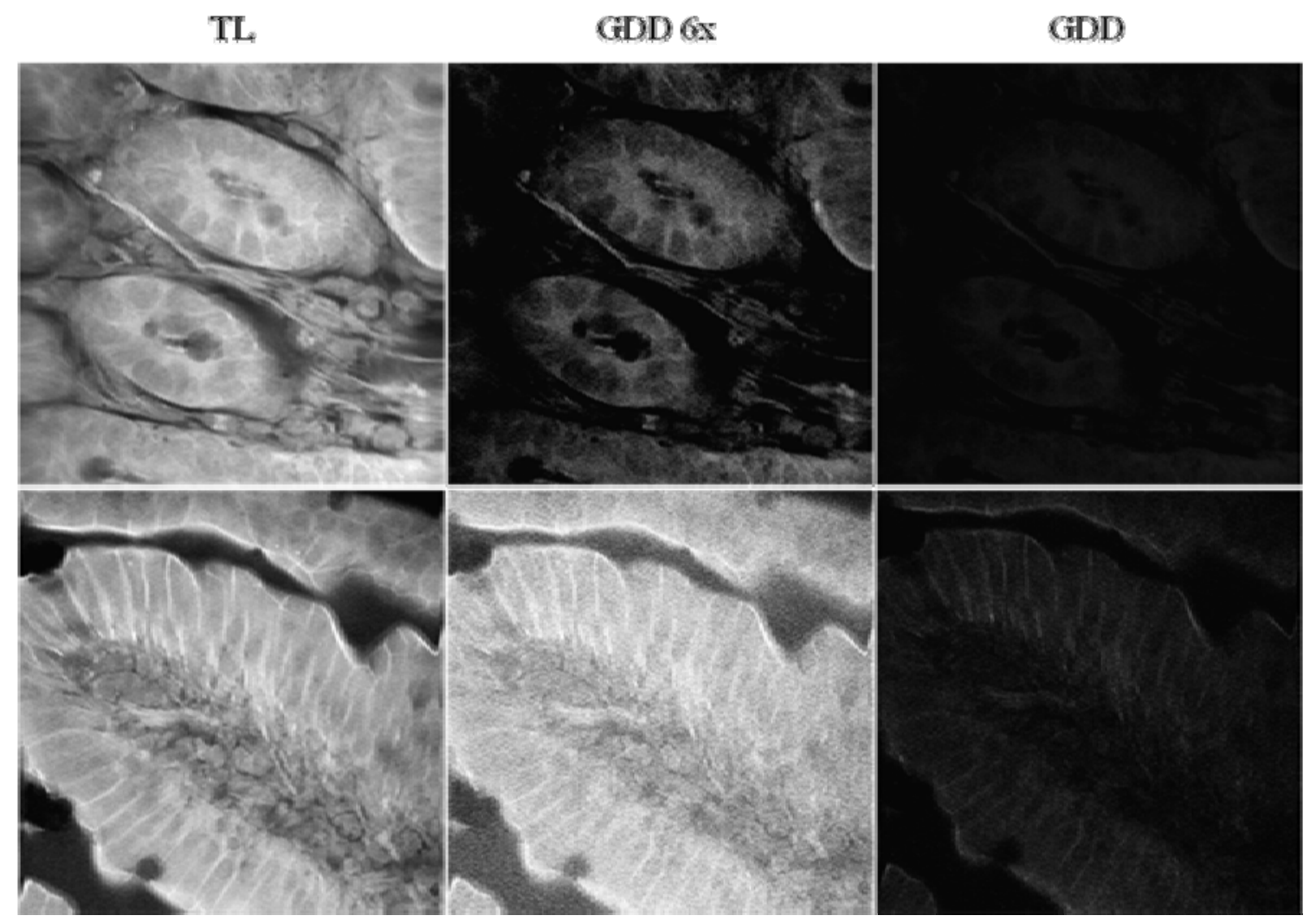

Fig. 3 


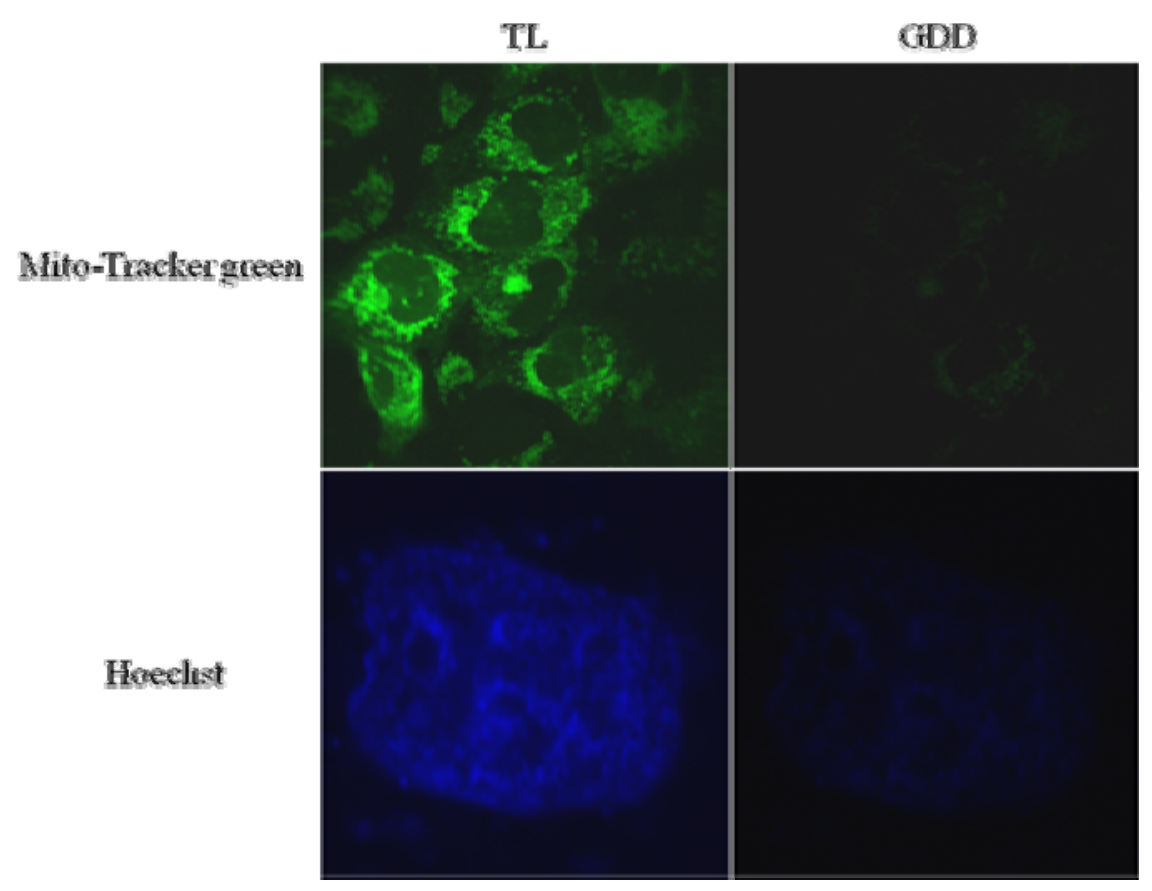

Fig. 4 


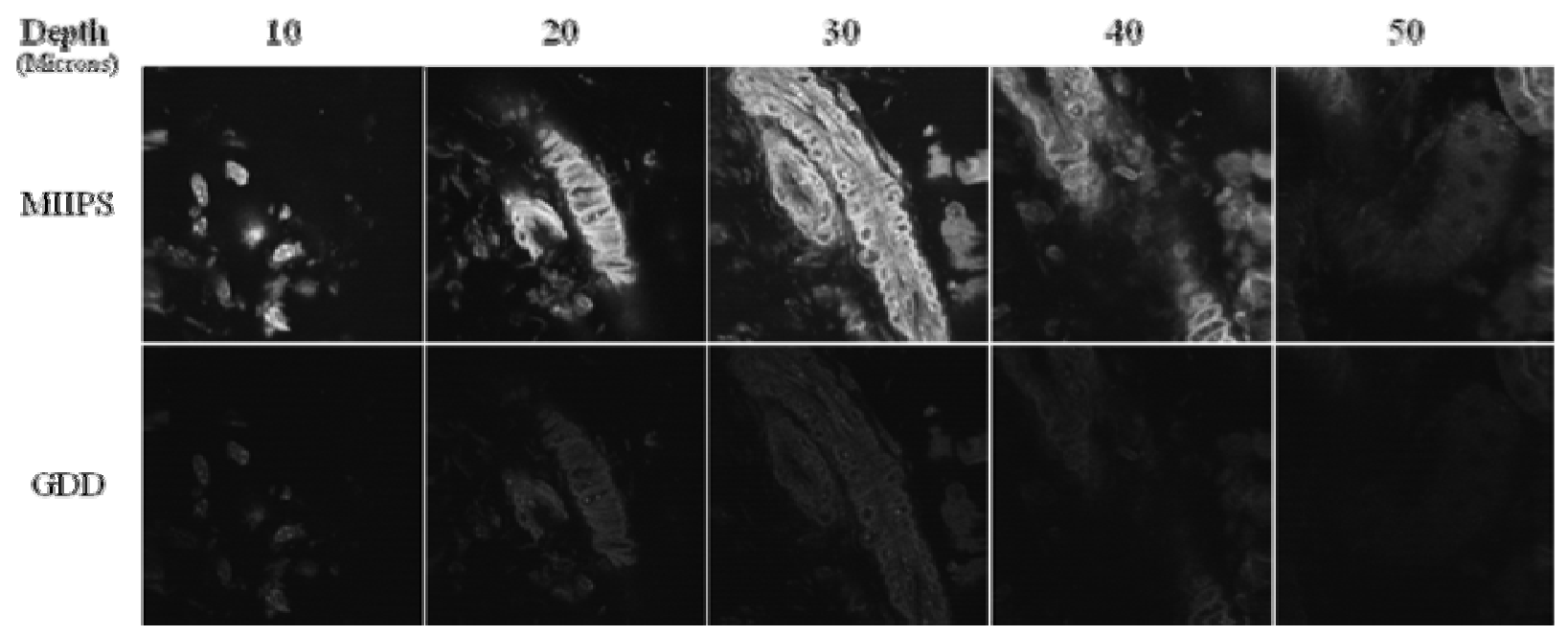

Fig. 5 


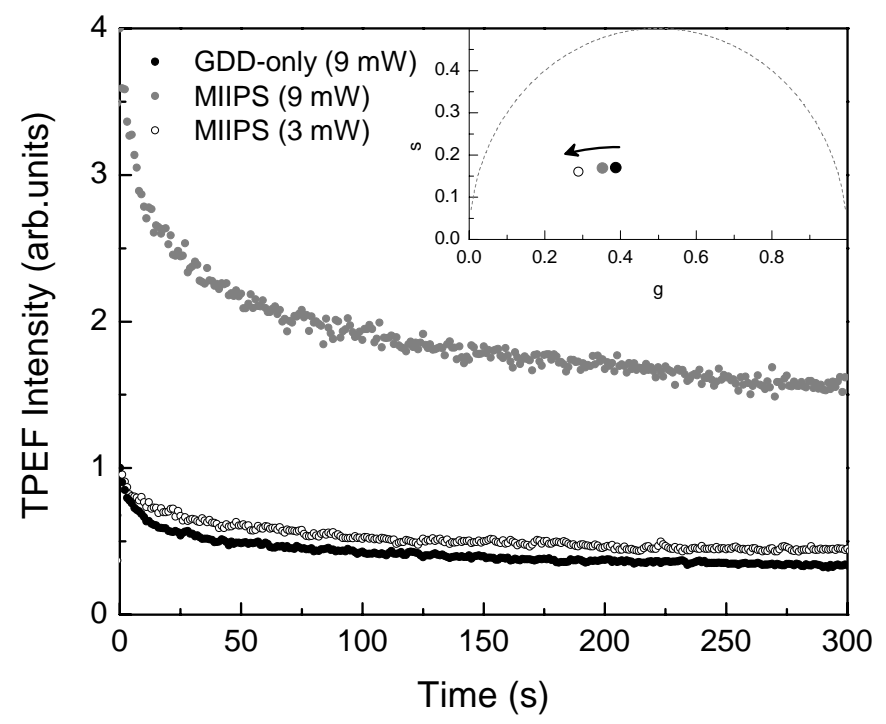

Fig. 6 


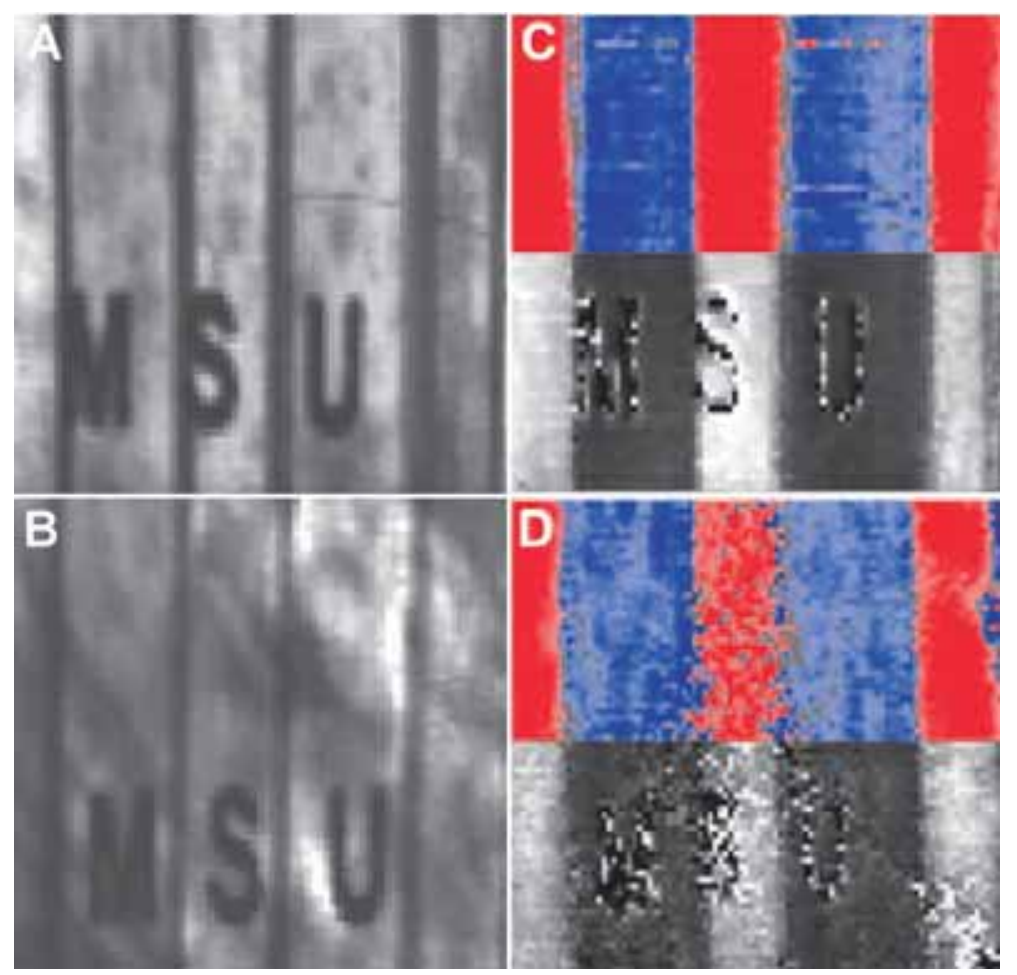

Fig. 7 


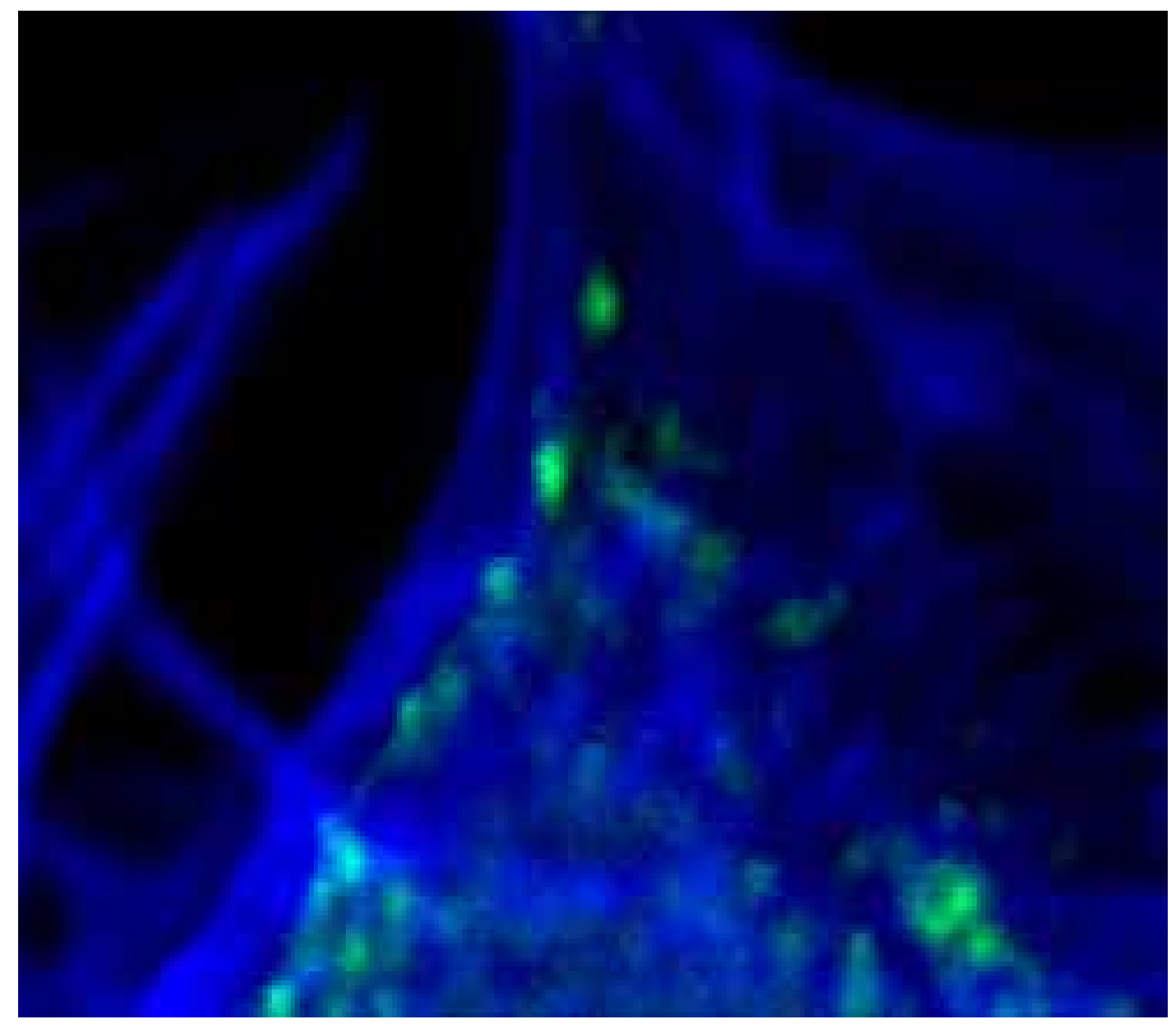

Fig. 8 


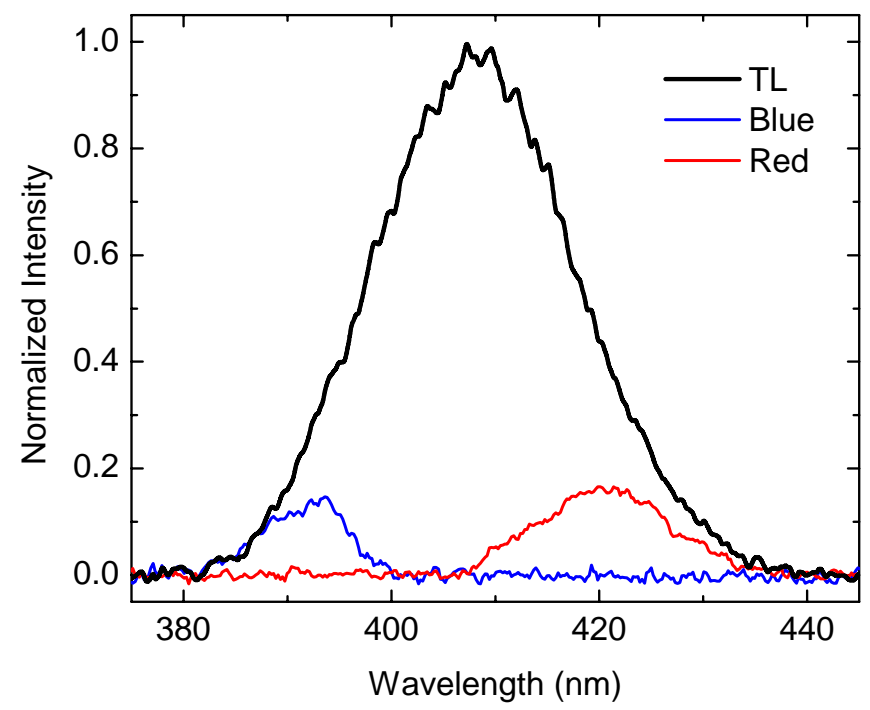

Fig. 9 